The study by Desai et al. included 42 patients with imatinib-resistant GISTs treated with sunitinib for at least 3 cycles and for an average of 37 weeks. All 42 patients had normal baseline thyroid function. Thyroid dysfunction was identified by measuring serum thyroid-stimulating hormone (TSH), and concentrations greater than $5.0 \mathrm{mU} / \mathrm{L}$ were considered abnormal. Overall, 26 (62\%) of the 42 patients had abnormal serum TSH concentrations after treatment and the authors reported that the risk for developing hypothyroidism increased with the duration of sunitinib therapy. The average time to hypothyroidism symptom presentation was 50 weeks and patients received L-thyroxine in order to normalize serum TSH concentrations.

On the basis of these observations, the authors conclude that the use of kinase inhibitors for the treatment of certain types of cancer can lead to undesirable side effects and that patients should be monitored for the incidence of such effects. More specifically, in the case of sunitinib-treated patients, clinicians should consider regular evaluation of serum TSH levels in order to treat patients who present with symptoms of hypothyroidism.

Original article Desai J et al. (2006) Hypothyroidism after sunitinib treatment for patients with gastrointestinal stromal tumors. Ann Intern Med 145: 660-664

\section{Thalidomide maintenance therapy improves survival in patients with multiple myeloma}

In patients with multiple myeloma, the average duration of response after chemotherapeutic protocols and autologous stem cell transplantation does not exceed 3 years; therefore, maintenance protocols that could prolong response and survival need to be established. A recent trial showed that thalidomide maintenance therapy is suitable for patients with multiple myeloma.

The trial randomized 597 patients with similar baseline characteristics to one of three treatment arms: no maintenance therapy $(n=200$; arm A), pamidronate only ( $n=196$; arm B) or pamidronate plus thalidomide $(n=201$; arm C). The percentage of patients with a complete or very good partial response was greater in arm C (67\%) than in arm A (55\%) or arm B (57\%), while overall survival 4 years after enrollment was $87 \%$ in arm C compared with $77 \%$ and
$74 \%$ for arms A and B, respectively. Moreover, patients treated with thalidomide had significantly improved event-free $(P=0.002)$, relapse-free $(P=0.003)$ and overall $(P=0.04)$ survival compared with non-thalidomidetreated patients. Thalidomide treatment was significantly beneficial for patients without a chromosome 13 deletion ( $P=0.006$ ) but not for patients with such a deletion $(P=0.2)$. Patients in the three arms had similar survival rates after relapse without skeletal events. In the thalidomide-treated group, however, certain drug-related adverse events were more prominent than in the nontreated groups, leading to discontinuation of thalidomide in $39 \%$ of the patients.

The authors suggest that thalidomide-based maintenance therapy after transplantation increases overall survival in multiple myeloma patients and could benefit patients who do not have a chromosome 13 deletion.

Original article Attal M et al. (2006) Maintenance therapy with thalidomide improves survival in patients with multiple myeloma. Blood 108: 3289-3294

\section{Improved PFS with rituximab maintenance in relapsed/ resistant follicular lymphoma}

Patients with follicular lymphoma almost always relapse following treatment, an event associated with poor median survival. Rituximab, an anti-CD20 monoclonal antibody, has a good pharmacokinetic and safety profile and has shown some success in this setting as monotherapy, as well as in combination with other therapies. Now, a phase III Intergroup trial has confirmed the ability of rituximab to improve both progression-free survival (PFS) and overall survival in patients with relapsed or resistant follicular lymphoma, when administered after CHOP (cyclophosphamide, doxorubicin, vincristine and prednisone) or $\mathrm{R}-\mathrm{CHOP}$ (CHOP plus rituximab) induction.

Initially, the trial randomized 465 patients to CHOP (6 cycles every 3 weeks) or R-CHOP (rituximab $375 \mathrm{mg} / \mathrm{m}^{2}$ administered intravenously on day 1). Overall and complete response were significantly improved with R-CHOP compared with $\mathrm{CHOP}$ induction $(85.1 \%$ vs $72.3 \%$ and $29.5 \%$ vs $15.6 \%$, respectively; $P<0.001$ for both), as was median PFS (33.1 months vs 20.2 months; $P<0.001$ ). In the next phase of the 